\title{
Evaluación de la concordancia entre dos métodos de lavado broncoalveolar para el diagnóstico microbiológico de la neumonía en pacientes con asistencia respiratoria mecánica
}

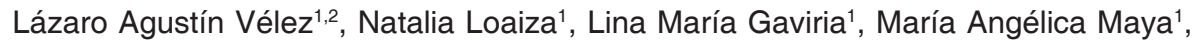 \\ Zulma Vanessa Rueda ${ }^{1}$, Luz Teresita Correa ${ }^{1}$, Jorge Ortega ${ }^{3}$, Héctor Ortega ${ }^{3}$ \\ 1 Grupo Investigador de Problemas en Enfermedades Infecciosas, Universidad de Antioquia, Medellín, \\ Colombia. \\ 2 Sección de Enfermedades Infecciosas, Departamento de Medicina Interna, Universidad de Antioquia- \\ Hospital Universitario San Vicente de Paúl, Medellín, Colombia. \\ ${ }^{3}$ Sección de Neumología, Departamento de Medicina Interna, Universidad de Antioquia-Hospital Universitario \\ San Vicente de Paúl, Medellín, Colombia. \\ Institución donde se realizó el trabajo: Hospital Universitario San Vicente de Paúl, Medellín, Colombia.
}

Introducción. El diagnóstico microbiológico de la neumonía permite optimizar el uso de antibióticos en pacientes con asistencia respiratoria mecánica. Para ello se han cultivado cuantitativamente las muestras del lavado broncoalveolar broncoscópico, procedimiento que no siempre es posible.

Objetivo. Evaluar la concordancia microbiológica entre muestras respiratorias tomadas por lavado broncoalveolar broncoscópico y no broncoscópico, y establecer si el uso previo de antibióticos y el momento de presentación de la neumonía pueden afectarla.

Materiales y métodos. Estudio prospectivo realizado en el Hospital Universitario San Vicente de Paúl, en 38 pacientes con sospecha de neumonía y con asistencia respiratoria mecánica. En todos se practicó el lavado broncoalveolar por fibrobroncoscopia y el lavado no broncoscópico usando un catéter telescopado de punta preformada (Balcath®). Todas las muestras fueron procesadas siguiendo protocolos microbiológicos convencionales.

Resultados. Considerando el lavado broncoalveolar por fibrobroncoscopia como patrón de referencia, los cultivos permitieron identificar el agente en $60,5 \%$ de los casos. El acuerdo diagnóstico se logró en $82 \%$ de los pacientes y $79 \%$ de los aislamientos. Utilizando el índice kappa de Cohen, la concordancia general entre los dos métodos fue 0,76 [0,60-0,93]; pero en quienes habían recibido antibióticos previos fue 0,26 [0,05-0,48], versus 1,0 en quienes no lo habían hecho $(p<0,0001)$. La concordancia no difirió significativamente cuando se compararon los casos de neumonía temprana y tardía.

Conclusiones. La concordancia general entre los dos métodos de lavado broncoalveolar es buena en pacientes con neumonía y respiración asistida mecánicamente. Sin embargo, el uso previo de antibióticos y no el momento de aparición de la neumonía, disminuye ésta significativamente.

Palabras clave: neumonía/etiología, respiración artificial, técnicas y procedimientos diagnósticos, técnicas microbiológicas, lavado broncoalveolar.

Concordance between two methods of bronchoalveolar lavage for the microbiological diagnosis of pneumonia in mechanically ventilated patients

Introduction. Microbiological diagnosis of pneumonia allows the optimal use of antibiotics in mechanically ventilated patients. That is why samples of bronchoscopic bronchoalveolar lavage had been quantitatively cultivated, but this procedure is not always possible.

Objective. To evaluate the microbiological concordance between respiratory samples obtained by non-bronchoscopic protected bronchoalveolar lavage compared to the bronchoscopic ones, and to find out whether concordance was affected by previous use of antibiotics or the time of pneumonia onset. 


\begin{abstract}
Materials and methods. Prospective study conducted at Hospital Universitario San Vicente de Paúl, in 38 patients with suspected pneumonia in mechanical ventilation. Bronchoalveolar lavage specimens were taken by two methods, the traditional one and non-bronchoscopic bronchoalveolar lavage, using a telescoping preformed tip catheter (Balcath®). All samples were processed using conventional microbiologic protocols.

Results. Considering flexible bronchoscopy with bronchoalveolar lavage as the gold standard, cultures allowed the identification of at least one respiratory pathogen in $60.5 \%$ of cases. Diagnostic agreement was achieved in $82 \%$ of patients and $79 \%$ of microbiologic isolates. Using the Cohen's kappa coefficient, general concordance between both methods was 0.76 [0.60-0.93]; but in those who received previously antibiotics was 0.26 [0.05-0.48], versus 1.0 in those who did not $(p<0.0001)$. Concordance did not differ significantly when cases of early or late pneumonia were compared.

Conclusions. Concordance between non-bronchoscopic and bronchoscopic bronchoalveolar lavage is good in mechanically ventilated patients with pneumonia. However, the use of antibiotics previously, but not the time of pneumonia presentation, significantly decreases that concordance.
\end{abstract}

Key words: pneumonia/etiology; respiration, artificial; diagnostic techniques and procedures, microbiological techniques, bronchoalveolar lavage.

La neumonía en pacientes sometidos a asistencia respiratoria mecánica invasiva causa altas tasas de morbilidad y mortalidad en las unidades de cuidado intensivo (1-4). Su diagnóstico microbiológico es clave para iniciar el tratamiento con antibióticos en forma racional y óptima, evitar la exposición innecesaria a ellos, muchos de ellos costosos y potencialmente tóxicos, y por lo tanto, disminuir el riesgo de aparición de bacterias resistentes (5).

El aspirado traqueobronquial ha sido, por su facilidad técnica y operativa, el procedimiento más usado para obtener secreciones respiratorias, a pesar de la pobre especificidad de los aislamientos microbiológicos así obtenidos. Lo anterior ha obligado a utilizar técnicas más invasivas, entre ellas el cultivo cuantitativo de muestras obtenidas de segmentos más profundos del pulmón, mediante el lavado broncoalveolar broncoscópico, el cual es considerado por muchos como la prueba de referencia para el diagnóstico microbiológico (5). Sin embargo, aunque controvertido, algunos

\section{Correspondencia:}

Lázaro Vélez, Grupo Investigador de Problemas en Enfermedades Infecciosas (GRIPE), Sede de Investigación Universitaria, Calle 62 № 52-59, laboratorio 630, Universidad de Antioquia, Medellín, Colombia.

Teléfono: (574) 219 6542; telefax: (574) 5129664.

clamona@une.net.co

Recibido: 19/05/08; aceptado:04/09/08 estudios sugieren que los resultados clínicos y el uso general de antibióticos utilizando el aspirado traqueobronquial, pueden ser similares a los obtenidos cuando se utiliza el lavado broncoalveolar (6).

Este último procedimiento requiere personal calificado, es costoso, no siempre está disponible $y$, ocasionalmente, no se puede realizar en pacientes inestables o con importante déficit respiratorio, por lo que se han desarrollado otros métodos para realizar el lavado broncoalveolar sin recurrir a la broncoscopia. La sencillez, fácil disponibilidad y rendimiento diagnóstico de estas técnicas, muy similares a los alcanzados con la prueba estándar (7-12), las han popularizado considerablemente. No obstante, estas técnicas no se han estandarizado por completo, la calidad de las muestras no ha sido investigada ampliamente y su reproducibilidad microbiológica, incluso en un mismo paciente, en general, sólo alcanza cifras alrededor del $75 \%$, especialmente cuando el paciente ha recibido antibióticos previamente (13).

Este estudio se llevó a cabo para evaluar en nuestro medio la utilidad diagnóstica de un método de lavado broncoalveolar no broncoscópico, usando un catéter protegido telescopado, comparada con la del lavado broncoalveolar tradicional. Nuestro objetivo primario fue determinar la concordancia general entre los 
diagnósticos microbiológicos de las muestras respiratorias obtenidas por ambos métodos y si dicha concordancia se modificaba de acuerdo con el tiempo de hospitalización en que se desarrolló la neumonía y el uso previo de antibióticos. Un segundo objetivo fue conocer los gérmenes responsables de la neumonía y su patrón de susceptibilidad según los factores anteriormente citados.

\section{Materiales y métodos}

\section{Pacientes}

Los adultos que recibían asistencia respiratoria mecánica invasiva en cualquiera de las tres unidades de cuidado intensivo (cardiovascular, médica o quirúrgica) del Hospital Universitario San Vicente de Paúl, en Medellín, Colombia, y que tuvieran sospecha de neumonía, entre septiembre de 2001 y noviembre de 2004, fueron evaluados por uno de los investigadores para su ingreso al estudio. Se incluyeron aquéllos mayores de 12 años en quienes se detectó la presencia de infiltrados pulmonares recientes que no pudieron ser atribuidos a otras causas, y dos o más de los siguientes tres hallazgos: i) temperatura mayor de $38^{\circ} \mathrm{C}$ o menor de $36^{\circ} \mathrm{C}$; ii) conteo de leucocitos mayor de 10.000 o menor de $4.000 / \mathrm{mm}^{3}$, o más del $10 \%$ de bandas; y iii) secreciones respiratorias purulentas. Todos los pacientes o sus familiares firmaron el consentimiento informado de acuerdo con los requerimientos legales (Ministerio de la Protección Social, Resolución 008430, 1993) y el estudio fue aprobado por el Comité de Ética de la Universidad de Antioquia y el Comité de Investigaciones del hospital. Ninguno de los investigadores intervino directamente en el tratamiento de los pacientes.

Los criterios de exclusión fueron la presencia de arritmias graves o inestabilidad hemodinámica que contraindicaran el procedimiento, hemoptisis masiva o riesgo mayor de sangrado, $\mathrm{PaO}_{2} / \mathrm{FiO}_{2}$ menor de 120, neutropenia menor de $500 / \mathrm{mm}^{3}$, sida, embarazo y haber recibido antibióticos en las últimas 72 horas, a no ser que la neumonía se hubiera desarrollado mientras se estaba recibiendo dicha terapia. Además, se excluyeron aquellos pacientes a los que no se les pudo tomar alguna de las dos muestras respiratorias que se analizaron en el estudio. Como el objetivo de éste era determinar la concordancia microbiológica y no la etiología de la neumonía asociada al respirador, se permitió la inclusión de pacientes que llevaran menos de 48 horas de asitencia respiratoria mecánica.

\section{Lavado broncoalveolar broncoscópico y no broncoscópico (Balcath®)}

Las muestras respiratorias de todos los pacientes se obtuvieron mediante lavado broncoalveolar. Éste se hizo por ambos métodos en forma consecutiva, bajo sedación con midazolam y fentanilo cuando la tos y la broncoconstricción asociada con el procedimiento así lo exigían. Primero se llevó a cabo el lavado broncoalveolar no broncoscópico, utilizando un catéter protegido telescopado, con extremo curvo preformado para poder dirigirlo hacia el pulmón afectado (BALCATH $囚$, Ballard Medical Products, Draper, Utah). Una vez colocado en el sitio donde se encontraba resistencia, se avanzaba el catéter interno hasta su acuñamiento y se instilaban 100 $\mathrm{ml}$ de solución salina isotónica estéril a través del catéter, distribuidos en alícuotas de $20 \mathrm{ml}$.

Inmediatamente después se realizaba el lavado broncoalveolar broncoscópico convencional, siguiendo las guías difundidas para tal fin (14). En resumen, el broncoscopio se avanzaba a través del tubo endotraqueal hasta acuñarlo en el subsegmento previamente elegido para la toma de la muestra de acuerdo con la distribución del compromiso evaluado por imágenes (radiografía, tomografía axial computadorizada o ambas). Sólo una vez acuñado, se aspiraban las secreciones tráqueo-bronquiales y se instilaban seis alícuotas de $20 \mathrm{ml}$ de solución salina estéril. En ambos procedimientos, el volumen instilado después de cada alícuota era inmediatamente recuperado por succión manual con jeringa; el de la primera alícuota se desechaba y el resto (entre 40 y 80 $\mathrm{ml}$ ) era enviado al laboratorio para su procesamiento.

\section{Procesamiento de las muestras}

Después de filtrar en gasa estéril y agitar con vórtex, las muestras se sembraron con asa calibrada para hacer cultivo bacteriano cuantitativo 
en agar sangre, agar chocolate suplementado y MacConkey. Todos los aislamientos con recuentos mayores o iguales a $10^{3}$ unidades formadoras de colonias (UFC/ml) fueron identificados hasta especie, siguiendo métodos convencionales. Las muestras del lavado broncoalveolar obtenidas por cualquiera de los dos métodos (broncoscópico o no) fueron consideradas de buena calidad si en el diferencial de las células del lavado el porcentaje de células epiteliales escamosas era menor de $1 \%$ (15).

Posteriormente, las muestras se centrifugaron a $4{ }^{\circ} \mathrm{C}$ y $4.500 \mathrm{rpm}$ durante 20 minutos; el sobrenadante fue descartado y el sedimento resuspendido en $1 \mathrm{ml}$ de agua destilada para lisar eritrocitos, y luego en $4 \mathrm{ml}$ de solución salina de Hank, agitando con vórtex tras cada adición. Después de ajustar su celularidad a $10^{5}$ células/ $\mathrm{ml}$, el resto de la muestra se citocentrifugó a 1.500 rpm por 15 minutos (Cytospin III, Shandon Instruments, Sewickley, PA). Las placas resultantes se colorearon con las tinciones de Wright y Gram, de acuerdo con el protocolo de Kahn-Jones (15).

\section{Variables analizadas}

Se recolectaron los datos clínicos y de laboratorio convencionales, con especial énfasis en la condición clínica de ingreso a la unidad de cuidados intensivos, criterios clínicos y radiológicos presentes para el diagnóstico de neumonía, días de hospitalización y de asistencia respiratoria mecánica al momento en que se tomaron las muestras respiratorias, análisis microbiológico de éstas, uso de antibióticos en la semana previa y condición al egreso (vivo o muerto).

\section{Criterios diagnósticos}

Al menos dos de los investigadores clínicos evaluaron la información en cada caso. Se definió como significativo cualquier aislamiento con un conteo bacteriano mayor o igual a $10^{4} \mathrm{UFC} / \mathrm{ml}$, cuando el paciente no había recibido antibióticos antes de tomar las muestras, o mayor o igual a $10^{3} \mathrm{UFC} / \mathrm{ml}$ si los había recibido $(16,17)$. La neumonía se consideró temprana cuando se desarrolló en los primeros cuatro días de ingreso al hospital, y tardía, cuando apareció después. Los aislamientos se definieron como resistentes cuando no eran susceptibles a los antibióticos que venía recibiendo el paciente y, en los casos en los que éstos no se administraron previamente, cuando el germen era resistente a los antimicrobianos considerados de primera elección para su tratamiento. Se consideró uso previo de antibióticos en quienes habían recibido antibióticos, al menos, 24 horas en la semana previa a la toma de las muestras respiratorias.

\section{Análisis estadístico}

El tamaño inicial de la muestra se calculó en 50 pacientes, por limitaciones estrictamente presupuestales. Los aislamientos de las muestras respiratorias obtenidas por fibrobroncoscopia fueron comparados con los aislamientos conseguidos por catéter sin fibrobroncoscopia para cada paciente.

Se determinó el porcentaje de acuerdo diagnóstico por el número de coincidencias sobre el número total de pacientes y de gérmenes. Éstos fueron considerados concordantes cuando la especie bacteriana y su antibiograma eran idénticos. Para todas las muestras se calculó la concordancia general y la obtenida según el tiempo de presentación de la neumonía y el uso previo de antibióticos. Se usó el índice kappa de Cohen como la medida de concordancia para los datos dicótomos producidos por las muestras obtenidas por ambos métodos. Para establecer si el uso previo de antibióticos y la presencia de neumonía tardía tuvieron alguna influencia sobre la presencia de resistencia en los gérmenes aislados, se construyeron tablas de contingencia de 2 por 2 para determinar la significación de las diferencias con la prueba exacta de Fisher o de ji al cuadrado con corrección de Yates, cuando fuera pertinente. El valor de $p<0,05$ fue considerado estadísticamente significativo. Para el manejo y análisis de los datos, usamos el programa SPSS $₫$, versión 15.0 (SPSS Inc; Chicago, Illinois) y Epidat $\AA$, versión 3.0 (Xunta de Galicia, Santiago de Compostela, España y Organización Panamericana de la Salud).

\section{Resultados}

\section{Pacientes}

Al final, por distintas dificultades de índole logística y administrativa, sólo pudieron incluirse 
38 pacientes hospitalizados en una cualquiera de las tres unidades de cuidado intensivo del hospital. El cuadro 1 muestra las características basales de los pacientes en el momento de ser incluidos en el estudio. La mediana de la edad fue de 40,5 años y la mayoría fueron hombres. Entre ellos, las condiciones clínicas que más frecuentemente explicaron el ingreso a la unidad de cuidados intensivos fueron de origen médico $(39,5 \%)$ y quirúrgico $(28,9 \%)$. Más de $75 \%$ de los pacientes tenía fiebre, secreciones respiratorias purulentas, leucocitosis y compromiso multilobar al inicio de la neumonía. Se presentaron más neumonías tardías que tempranas, y la mitad de los pacientes había recibido antibióticos en la semana previa a la toma de las muestras. Seis de los 38 pacientes incluidos $(15,8 \%)$ llevaban menos de 48 horas en asistencia respiratoria mecánica; el resto cumplía con la definición de neumonía asociada al respirador.

\section{Etiología}

Se obtuvieron 34 aislamientos, 33 por fibrobroncoscopia y 32 por catéter sin fibrobroncoscopia. Considerando la fibrobroncoscopia como patrón de referencia, los cultivos fueron negativos en 15 de los 38 pacientes estudiados $(39,5 \%)$, y polimicrobianos en $7(18,4 \%)$. El cuadro 2 muestra los hallazgos microbiológicos en el lavado broncoalveolar de las muestras tomadas por fibrobroncoscopia de acuerdo con el momento de presentación de la neumonía y el uso previo de antibióticos. Los gérmenes más frecuentemente aislados fueron Staphylococcus aureus (30,3\%)

Cuadro 1. Características basales de 38 pacientes con neumonía sometidos a asistencia respiratoria mecánica.

\begin{tabular}{|c|c|}
\hline Variable & Valor \\
\hline $\begin{array}{l}\text { Edad (años), promedio } \pm \mathrm{DE}^{\mathrm{i}} \text { (rango) } \\
\text { Hombre/mujer, } \mathrm{n}(\%)\end{array}$ & $\begin{array}{l}40,3 \pm 18,2(13-84) \\
26(68,4): 12(31,6)\end{array}$ \\
\hline $\begin{array}{l}\text { Condición clínica de ingreso } \\
\text { - Médica, n (\%) } \\
\text { - Traumática, n (\%) } \\
\text { - Quirúrgica, n (\%) } \\
\text { - Toxicológica, n (\%) }\end{array}$ & $\begin{array}{l}15(39,4) \\
9(23,6) \\
11(28,9) \\
3(7,8)\end{array}$ \\
\hline $\begin{array}{l}\text { Temperatura en }{ }^{\circ} \mathrm{C} \text {, promedio } \pm \mathrm{DE} \text { (rango) } \\
\cdot<36^{\circ} \mathrm{C}, \mathrm{n}(\%) \\
\cdot>38^{\circ} \mathrm{C}, \mathrm{n}(\%)\end{array}$ & $\begin{array}{l}38,4 \pm 1,0(35-40) \\
3(7,8) \\
33(86,8)\end{array}$ \\
\hline Secreciones respiratorias purulentas, sí/no, n (\%) & $37(97,4): 1(2,6)$ \\
\hline $\begin{array}{l}\text { Leucocitos, promedio } / \mathrm{mm}^{3} \pm \mathrm{DE} \text { (rango) } \\
\cdot<4.000 / \mathrm{mm}^{3}, \mathrm{n}(\%) \\
\cdot>10.000 / \mathrm{mm}^{3}, \mathrm{n}(\%)\end{array}$ & $\begin{array}{l}14.676 \pm 6.326(3.700-29.400) \\
1(2,6) \\
29(76,3)\end{array}$ \\
\hline $\begin{array}{l}\text { Hallazgos radiológicos } \\
\text { - Compromiso multilobar, n (\%) } \\
\text { - Infiltrados alveolares, n (\%) } \\
\text { - Derrame pleural, n (\%) }\end{array}$ & $\begin{array}{l}30(78,9) \\
33(86,8) \\
18(47,4)\end{array}$ \\
\hline Uso previo de antibióticos, $\mathrm{n}(\%)$ & $19(50)$ \\
\hline $\begin{array}{l}\text { Tipo de neumonía } \\
\text { - Temprana, n (\%) } \\
\text { - Tardía, n (\%) }\end{array}$ & $\begin{array}{l}15(39,5) \\
23(60,5)\end{array}$ \\
\hline $\begin{array}{l}\text { Tiempo en el que se desarrolló la neumonía: } \\
\text { - Estancia hospitalaria en días, promedio } \pm \text { DE (rango) } \\
\text { - Estancia en UCli en días, promedio } \pm \text { DE (rango) } \\
\text { - Días de RMA } A^{i i i} \text {, promedio } \pm \text { DE (rango) }\end{array}$ & $\begin{array}{l}10,0 \pm 8,1(3-44) \\
7,2 \pm 4,6(2-23) \\
6,6 \pm 4,2(0-17)\end{array}$ \\
\hline
\end{tabular}

' Desviación estándar

i Unidad de Cuidado Intensivo

ii Respiración mecánica asistida 
Cuadro 2. Hallazgos microbiológicos en el lavado broncoalveolar de las muestras tomadas por fibrobroncoscopia en 38 casos de neumonía de acuerdo con el tiempo de presentación y uso previo de antibióticos.

\begin{tabular}{|c|c|c|c|c|c|}
\hline Hallazgo microbiológico & $\begin{array}{c}\text { Neumonía } \\
\text { temprana }\end{array}$ & $\begin{array}{c}\text { Neumonía } \\
\text { tardía (\%) }\end{array}$ & $\begin{array}{l}\text { Sin uso previo } \\
\text { de antibióticos }\end{array}$ & $\begin{array}{l}\text { Con uso previo } \\
\text { de antibióticos (\%) }\end{array}$ & Total $(\%)$ \\
\hline Cultivo negativo, número de pacientes & 5 & $10(67)$ & 7 & $8(53)$ & $15(39,5)$ \\
\hline Cultivo positivo, número de pacientes & 10 & $13(57)$ & 12 & $11(48)$ & $23(60,5)$ \\
\hline $\begin{array}{c}\text { - Staphylococcus aureus } \\
\cdot \text { MRSA }^{\varepsilon} \\
\cdot \text { MSSA }^{¥}\end{array}$ & $\begin{array}{l}2 \\
2\end{array}$ & $\begin{array}{l}4(67) \\
2(50)\end{array}$ & $\begin{array}{l}2 \\
4\end{array}$ & $\begin{array}{l}4(67) \\
0(0)\end{array}$ & $10(30,3)$ \\
\hline $\begin{aligned} \text { - BGN no fermentadores } \\
\cdot \text { P. aeruginosa } \\
\cdot \text { Acinetobacter spp. } \\
\cdot \text { Otros }^{i}\end{aligned}$ & $\begin{array}{l}0 \\
1 \\
1\end{array}$ & $\begin{array}{l}3(100) \\
1(50) \\
3(75)\end{array}$ & $\begin{array}{l}0 \\
0 \\
1\end{array}$ & $\begin{array}{l}3(100) \\
1(50) \\
3(75)\end{array}$ & $9(27,3)$ \\
\hline $\begin{array}{l}\text { - Haemophilus influenzae } \\
\text { - Enterobacterias } \\
\cdot \text { K. pneumoniae } \\
\cdot \text { Otrosii }\end{array}$ & $\begin{array}{l}0 \\
1\end{array}$ & $\begin{array}{l}1(17) \\
3(100) \\
1(50)\end{array}$ & $\begin{array}{l}1 \\
1\end{array}$ & $\begin{array}{l}0(0) \\
2(67) \\
1(50)\end{array}$ & $\begin{array}{l}6(18,2) \\
5(15,2)\end{array}$ \\
\hline - Otros ${ }^{\mathrm{iii}}$ & 2 & $1(33)$ & 3 & $0(0)$ & $3(9,1)$ \\
\hline Total aislamientos bacterianos & 14 & $19(58)$ & 19 & $14(42)$ & $33(100)$ \\
\hline
\end{tabular}

y bacilos Gram negativos no fermentadores (27,3\%). Estos últimos y S. aureus, en general, resistente a la meticilina, estuvieron especialmente presentes en los casos de neumonía tardía y uso previo de antibióticos. Todos los casos de $S$. aureus sensible a la meticilina y Haemophilus influenzae se presentaron en pacientes que no habían recibido tratamiento antibacteriano.

\section{Acuerdo diagnóstico}

El cuadro 3 muestra el porcentaje de acuerdo diagnóstico entre los resultados microbiológicos de las muestras tomadas por las dos técnicas usadas, tanto por número de pacientes como por número de gérmenes aislados. Se logró acuerdo microbiológico en 31 de los 38 pacientes estudiados $(81,6 \%)$ y 27 de los $34(79,4 \%)$ aislamientos logrados por ambos métodos fueron idénticos. No hubo diferencias significativas en el acuerdo diagnóstico entre los diferentes gérmenes. Sólo una de las muestras de lavado broncoalveolar tomadas por fibrobroncoscopia y dos de las tomadas por catéter sin fibrobroncoscopia tenían un porcentaje de células epiteliales escamosas mayor o igual a 1\%. En ninguno de los casos hubo acuerdo diagnóstico entre ambas muestras.

\section{Concordancia}

La figura 1 ilustra la concordancia obtenida entre la fibrobroncoscopia y el método con catéter, de acuerdo con el tipo de neumonía y el uso previo de antibióticos. El índice kappa de Cohen para evaluar la concordancia general entre los dos métodos fue 0,7648 $\pm 0,0845$ [IC95\% 0,5991$0,9305]$. Cuando se analizó en los pacientes que habían recibido antibióticos previamente, el índice fue de 0,2630 $\pm 0,1090[0,0495-0,4766]$, mientras que fue de 1,0 en quienes no habían recibido antibióticos $(p<0,0001)$. En los casos de neumonía temprana y tardía, la concordancia no difirió significativamente (índices kappa de 0,7119 $\pm 0,1219[0,4729-0,9508]$ y $0,6815 \pm 0,1144$ [0,4574-0,9057], respectivamente). 
Cuadro 3. Porcentaje de acuerdo diagnóstico por pacientes y gérmenes entre las muestras tomadas por fibrobroncoscopia y con catéter (BALCATH®) en 38 casos de neumonía en pacientes sometidos a asistencia respiratoria mecánica.

\begin{tabular}{|c|c|c|c|}
\hline Variable & $\begin{array}{c}\text { Fibrobroncoscopio } \\
(n=38)\end{array}$ & $\begin{array}{c}\text { Balcath }{ }^{(n=38)} \\
\text { (n) }\end{array}$ & $\begin{array}{l}\text { Porcentaje } \\
\text { de acuerdo }\end{array}$ \\
\hline Cultivos negativos, número de pacientes & 15 & 15 & 13 de $15(86,6)$ \\
\hline Cultivos monomicrobianos, número de pacientes & 16 & 17 & 12 de $16(75)$ \\
\hline Cultivos polimicrobianos, número de pacientes & 7 & 6 & 6 de $7(85,7)$ \\
\hline Staphylococcus aureus & 10 & 10 & 8 de $10(80)$ \\
\hline BGN no fermentadoresi & 9 & 8 & 6 de $9(66,6)$ \\
\hline Haemophilus influenzae & 6 & 6 & 6 de $6(100)$ \\
\hline Enterobacteriasii & 5 & 5 & 4 de $5(80)$ \\
\hline Otrosiii & 3 & 3 & 3 de $3(100)$ \\
\hline Total aislamientos bacterianos & 33 & 32 & 27 de $34(79,4)$ \\
\hline
\end{tabular}

' Bacilos Gram negativos no fermentadores aislados: Pseudomonas aeruginosa, Alcaligenes xylosoxidans, Acinetobacter baumannii, Acinetobacter iwoffi, Burkholderia cepacia, Stenotrophomonas maltophilia.

' Enterobacterias aisladas: Klebsiella pneumoniae, Escherichia coli, Proteus mirabilis, Enterobacter cloacae

ii Otros aislamientos: Moraxella catarrhalis, Enterococcus spp., Streptococcus pneumoniae

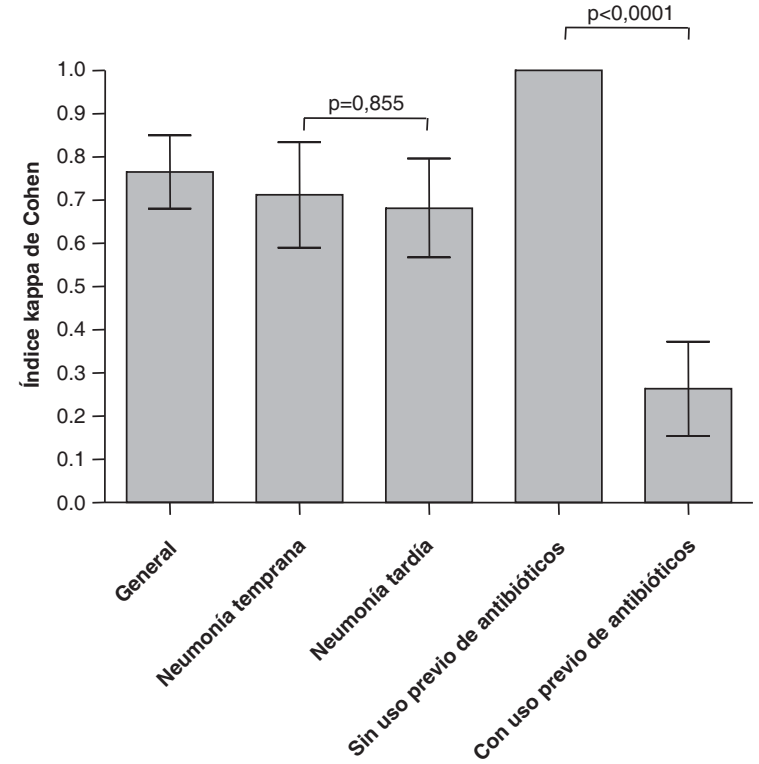

Figura 1. Concordancia en el diagnóstico microbiológico entre las muestras del lavado broncoalveolar tomadas por fibrobroncoscopia y por catéter (Balcath®) de acuerdo con el tipo de neumonía y el uso previo de antibióticos.

Patrones de susceptibilidad de los
asilamientos obtenidos por fibrobroncoscopia

La figura 2 ilustra el patrón de susceptibilidad de los gérmenes aislados en muestras tomadas por fibrobroncoscopia de acuerdo con el tiempo de desarrollo de la neumonía y el uso previo de antibióticos. En las 38 muestras tomadas por este método se aislaron 33 bacterias, 30 de ellas con antibiograma disponible. En general, y de acuerdo con la definición enunciada anteriormente, el $60 \%$ se consideró resistente (18/30). Cuando se analizó dicha resistencia en los pacientes que habían recibido antibióticos en la semana previa a la toma de las muestras, 13 de los 14 gérmenes aislados $(92,8 \%)$ se consideraron resistentes, versus 5 de $16(31,2 \%)$ en quienes no los habían recibido $(p=0,0008, O R=28,6[2,9-283,1])$. Entre los pacientes que desarrollaron neumonía tardía se obtuvieron 16 aislamientos, 13 de los cuales fueron resistentes $(81,2 \%)$, versus 5 de $14(35,7 \%)$ en los pacientes con neumonía temprana $(\mathrm{p}=0,02$, $\mathrm{OR}=7,8[1,5-41,2])$.

\section{Mortalidad}

Durante la estancia hospitalaria murieron 14 de los 38 pacientes estudiados $(36,8 \%)$. No hubo diferencias significativas en la mortalidad según el tiempo de presentación de la neumonía, 33,3\% $(5 / 15)$ en la temprana y $39,1 \%(9 / 23)$ en la tardía, o el uso previo de antibióticos, $42,1 \%(8 / 19)$ en quienes los habían usado y $31,6 \%(6 / 19)$ en quienes no lo habían hecho.

\section{Discusión}

Nuestros resultados demuestran que el uso previo de antibióticos disminuye significativamente la concordancia existente entre la fibrobroncoscopia 


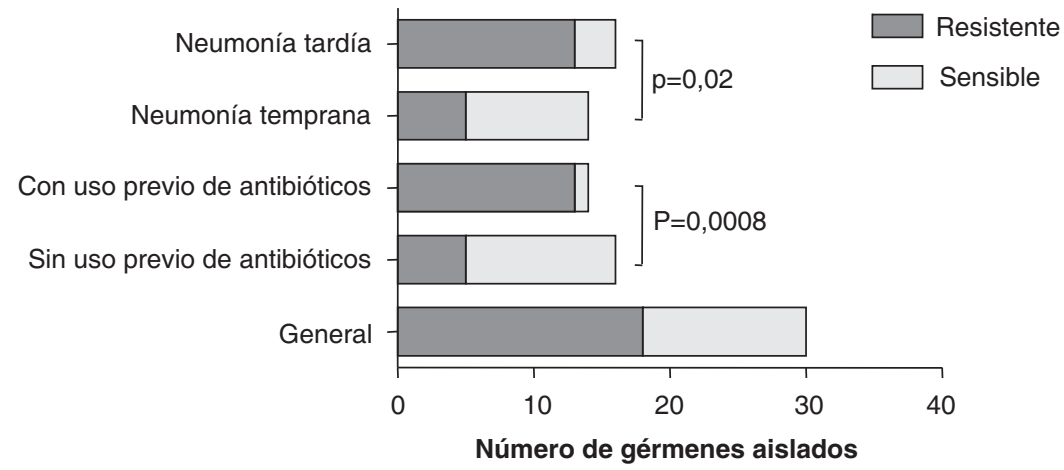

Figura 2. Patrón de susceptibilidad de 30 gérmenes aislados en muestras tomadas por fibrobroncoscopia de acuerdo con el tipo de neumonía y el uso previo de antibióticos.

y el método por catéter para diagnosticar el agente etiológico en las secreciones respiratorias de los pacientes con asistencia respiratoria mecánica con neumonía. Dicha concordancia no difiere entre los casos de neumonía temprana y tardía, lo cual sugiere que cualquier diferencia existente en estos casos depende más del uso previo de antibióticos que del tiempo de presentación de la neumonía y, por tanto, del tipo de gérmenes asociados con cada uno de estos escenarios clínicos.

Aunque la eficacia de los métodos broncoscópicos y no broncoscópicos para establecer la etiología microbiana en los pacientes con neumonía sometidos a asistencia respiratoria mecánica es aceptada por la mayoría de los autores $(5,11,12,18$ $20)$, algunos creen que las técnicas no broncoscópicas todavía deben ser validadas (16). En la literatura revisada, al menos 12 estudios evaluaron, en 552 pacientes con sospecha de neumonía, el acuerdo diagnóstico, la concordancia microbiológica o ambas, existentes entre algunos métodos broncoscópicos y no broncoscópicos. En 10 de ellos se comparó el cepillado protegido, ciego, con el broncoscópico (8,21-29), en cuatro, el cepillado protegido ciego con el lavado broncoalveolar broncoscópico $(23-25,28)$, en dos, el lavado broncoalveolar no broncoscópico con el cepillado broncoscópico $(22,30)$, y en sólo uno, el lavado broncoalveolar broncoscópico con el lavado broncoalveolar no broncoscópico (31).

En general, el acuerdo diagnóstico osciló entre $61 \%$ y $100 \%$, mientras que la concordancia, en los cuatro trabajos que la describen $(22,25,28,30)$, fluctuó entre 0,55 y 1,0. Algunos autores también han comparado dos técnicas broncoscópicas entre sí, el cepillado protegido con el lavado broncoalveolar $(23,25,26,28,32-34)$, y dos pruebas ciegas, el cepillado protegido con el lavado broncoalveolar, ambos no broncoscópicos (35). No se encontraron diferencias significativas entre las técnicas empleadas. No obstante, es de resaltar que el acuerdo y la concordancia más bajos $(52,2 \%$ y 0,46 , respectivamente) se obtuvieron en dos estudios que cotejaron el cepillado y el lavado broncoalveolar broncoscópicos $(28,32)$. Aunque no es clara la razón por la cual la concordancia varía tanto entre los diferentes estudios, todo sugiere diferencias metodológicas. Independientemente de ellas, nuestros resultados generales promedian los rangos descritos arriba.

Sin embargo, a diferencia de nuestro estudio, pocos autores evalúan las variables que afectan negativamente la concordancia, como el uso previo de antibióticos y el momento de presentación de la neumonía. La mayoría sólo se limita a describir la disminución de la exactitud diagnóstica de las distintas técnicas usadas cuando los pacientes han recibido antibióticos recientemente $(17,23,24,29,33,35-38)$. Sólo Bello y Timsit $(23,32)$ evaluaron el acuerdo diagnóstico en estas circunstancias, ambos entre dos técnicas broncoscópicas, y encontraron resultados aparentemente contradictorios. El primero reportó 
que el acuerdo diagnóstico fue superior en el grupo que no había recibido antibióticos (10\%-52\% mayor), pero el segundo no encontró diferencias significativas entre ambos grupos (acuerdo de $57,9 \%$ versus $52,2 \%$ en pacientes con antibióticos y sin ellos, respectivamente); es necesario aclarar, sin embargo, que en este último estudio el tratamiento antibiótico fue dirigido a otro foco infeccioso, e iniciado antes de cualquier signo de neumonía.

Es de suponer que, cuando los pacientes han recibido antibióticos previamente, la disminución de la exactitud diagnóstica y de la concordancia microbiológica entre las técnicas usadas para el diagnóstico etiológico de la neumonía, se deba a la disminución de varios órdenes de magnitud en el recuento bacteriano de los gérmenes causantes de la neumonía, y a la mayor probabilidad de aislar microorganismos que sólo están colonizando el tracto respiratorio, un fenómeno semejante a lo que ocurre cuando se evalúa la reproducibilidad de cualquier técnica en pacientes que ya han recibido antibióticos previamente.

A diferencia de lo anterior, es ampliamente reconocido el impacto de los antibióticos sobre el aislamiento de gérmenes resistentes (39-41) y nuestro estudio no fue ajeno a ello. En los pacientes que no los habían usado, se aislaron especialmente $S$. aureus, principalmente sensible a la meticilina, y $H$. influenzae. Cuando se habían utilizado, $S$. aureus resistente a la meticilina y los bacilos Gram negativos, ambientales o entéricos, explicaron la mayor parte de los casos. Estos resultados coinciden con los de Rello y colaboradores (42), quienes, además, encontraron, y a diferencia de nosotros, que el uso previo de antibióticos fue el único factor independientemente asociado con mortalidad, posiblemente debido a la selección de organismos más letales.

El momento de aparición de la neumonía es otro factor asociado con el aislamiento de gérmenes resistentes. Las tardías son causadas principalmente por bacterias resistentes, en la medida en que la presión selectiva ejercida por antibióticos en el ambiente hospitalario facilita la colonización, y posteriormente la enfermedad debida a ellas $(43,44)$. En nuestro estudio, sin embargo, pudo observarse la presencia de bacterias resistentes en neumonías tempranas y de gérmenes sensibles en las tardías, lo que sugiere que el aislamiento de bacterias resistentes depende más del uso previo de antibióticos que del tiempo de presentación de la neumonía.

Nuestro estudio tiene varias limitaciones. Entre ellas es necesario mencionar el bajo número de pacientes finalmente incluidos en el estudio y la falta de estandarización en relación con el uso de antibióticos, esta última explicable porque los investigadores no intervinieron directamente en el manejo de los pacientes. Sin embargo, los resultados obtenidos proporcionan datos muy útiles que ayudan a incrementar la certeza del diagnóstico microbiológico, el cual es fundamental para usar más racionalmente los antibióticos y para manejar adecuadamente los pacientes en asistencia respiratoria mecánica con neumonía. La otra limitación, compartida por todos los estudios de este tipo, es la ausencia de un método de referencia para el diagnóstico microbiológico de la neumonía asociada al respirador. Sin embargo, nuestro estudio pretendía comparar microbiológicamente dos métodos diagnósticos entre sí, uno de los cuales, el lavado broncoalveolar broncoscópico, es el más ampliamente aceptado para este fin.

En conclusión, los resultados demuestran que el lavado broncoalveolar no broncoscópico utilizado en nuestro estudio tiene buena concordancia microbiológica con el lavado broncoalveolar broncoscópico tradicional. Éste es un procedimiento rápido, seguro y de fácil realización por médicos, enfermeros o terapeutas respiratorios previamente capacitados. En tal sentido, permite la toma inmediata de muestras respiratorias en el paciente con asistencia respiratoria mecánica tan pronto se le hace el diagnóstico de neumonía, a cualquier hora del día o de la noche, antes del inicio de los antibióticos y sin exigir la disponibilidad de un endoscopio ni la presencia de un neumólogo en la unidad de cuidado intensivo. Sin embargo, debido a la baja concordancia en pacientes previamente tratados con antibióticos, en ellos es necesario recurrir al lavado broncoalveolar broncoscópico tradicional, método considerado de referencia en el diagnóstico microbiológico de estos casos. 


\section{Conflicto de intereses}

Lázaro Vélez ha recibido financiación para investigación de AstraZeneca y Roche Colombia, y ha sido consultor de Pfizer. Los otros autores no declaran conflicto de intereses.

\section{Financiación}

Este estudio fue patrocinado por la Universidad de Antioquia, a través del CODI; AstraZeneca financió los catéteres Balcath®.

\section{Referencias}

1. Heyland DK, Cook DJ, Griffith L, Keenan SP, BrunBuisson C. The attributable morbidity and mortality of ventilator-associated pneumonia in the critically ill patient. The Canadian Critical Trials Group. Am J Respir Crit Care Med. 1999;159:1249-56.

2. Bercault $\mathbf{N}$, BoulainT. Mortality rate attributable to ventilator-associated nosocomial pneumonia in an adult intensive care unit: a prospective casecontrol study. Crit Care Med. 2001;29:2303-9.

3. Fagon JY, Chastre AJ, Hance AJ, Montravers P, Novara A, Gilbert C. Nosocomial pneumonia in ventilated patients: a cohort study evaluating attributable mortality and hospital stay. Am J Med. 1993;94:281-8.

4. Chastre J, Fagon JY. Ventilator-associated pneumonia. Am J Respir Crit Care Med. 2002;165:867-903.

5. Fagon JY, Chastre J, Wolff M, Gervais C, ParerAubas S, Stéphan F, et al. Invasive and non-invasive strategies for management of suspected ventilator associated pneumonia: a randomized trial. Ann Intern Med. 2000;132:621-30.

6. The Canadian Critical Care Trials Group. A randomized trial of diagnostic techniques for ventilator-associated pneumonia. N Engl J Med. 2006;21:2619-30.

7. Torres A, El-Ebiary M, Padró L, González J, de la Bellacasa JP, Ramírez J. Validation of different techniques for the diagnosis of ventilator-associated pneumonia. Comparison with immediate postmortem pulmonary biopsy. Am J Respir Crit Care Med.1994; 149:324-31

8. Marik P, Brown W. A comparison of bronchoscopic $V s$. blind protected specimen brush sampling in patients with suspected ventilator-associated pneumonia. Chest. 1995;108:203-7.

9. Torres A, El-Ebiary M. Invasive diagnostic techniques for pneumonia: protected specimen brush, bronchoalveolar lavage and lung biopsy methods. Infect Dis Clin North Am. 1998;12;701-22.

10. Pittet D, Harbarth S. What techniques for diagnosis of ventilator-associated pneumonia? Lancet. 1998; 352:83-4.
11. Casetta M, Blot F, Antoun S, Leclercq B, Tancrède C, Doyon F, et al. Diagnosis of nosocomial pneumonia in cancer patients undergoing mechanical ventilation: a prospective comparison of the plugged telescoping catheter with the protected specimen brush. Chest. 1999;115:1641-5.

12. Heyland DK, Cook DJ, Marshall J, Heule M, Guslits $\mathrm{B}$, Lang $\mathrm{J}$, et al. The clinical utility of invasive diagnostic techniques in the setting of ventilator-associated pneumonia. Canadian Care Trials Group. Chest. 1999;115:1076-84.

13. Grossman R, Fein A. Evidence-based assessment of diagnostic test for ventilator associated pneumonia: executive summary. Chest. 2000;117:177S-81.

14. American Thoracic Society. Clinical role of bronchoalveolar lavage in adults with pulmonary disease. Am J Respir Crit Care Med. 1990;142:481-86.

15. Kahn FW, Jones JM. Analysis of bronchoalveolar lavage specimens from immunocompromised patients with a protocol applicable in the microbiology laboratory. $J$ Clin Microbiol. 1988;26:1150-5.

16. Mayhall CG. Ventilator-associated pneumonia or not? Contemporary diagnosis. Emerg Infect Dis. 2001;7:200-4.

17. Souweine B, Veber B, Bedos JP, Gachot B, Dombret MC, Regnier B, et al. Diagnostic accuracy of protected specimen brush and bronchoalveolar lavage in nosocomial pneumonia: Impact of previous antimicrobial treatments. Crit Care Med. 1998;26:236-44.

18. Campbell GD. Blinded invasive diagnostic procedures in ventilator-associated pneumonia. Chest. 2000;117:207S-11.

19. Woske HG, Röding T, Schulz I, Lode H. Ventilatorassociated pneumonia in a surgical intensive care unit: epidemiology, etiology and comparison of three bronchoscopic methods for microbiological specimen sampling. Crit Care. 2001;5:167-73.

20. Chastre J, Fagon JY, Bornet-Lecso M, Calvat S, Dombret MC, al Khani R, et al. Evaluation of bronchoscopic techniques for the diagnosis of nosocomial pneumonia. Am J Respir Crit Care Med. 1995;152:231-40.

21. Pham LH, Brun-Buisson C, Legrand $P$, Rauss $A$, Verra F, Brochard L, et al. Diagnosis of nosocomial pneumonia in mechanically ventilated patients. Comparison of a plugged telescoping catheter with the protected specimen brush. Am Rev Respir Dis. 1991;143:1055-61.

22. Wearden PD, Chendrasekhar A, Timberlake GA. Comparison of nonbronchoscopic techniques with bronchoscopic brushing in the diagnosis of ventilatorassociated pneumonia. J Trauma. 1996;41:703-7.

23. Bello S, Tejada A, Chacón E, Villuendas MC, Senar A, Gascon M, et al. "Blind" protected specimen brush- 
ing versus bronchoscopic techniques in the aetiolological diagnosis of ventilator-associated pneumonia. Eur Respir J. 1996;9:1494-9.

24. Jordá R, Parras F, Ibañez J, Reina J, Bregada J, Raurich JM. Diagnosis of nosocomial pneumonia in mechanically ventilated patients by the blind protected telescoping catheter. Intensive Care Med. 1993; 19: 377-82.

25. Papazian L, Martin C, Meric B, Dumon JF, Gouin F. A reappraisal of blind bronchial sampling in the microbiologic diagnosis of nosocomial bronchopneumonia. A comparative study in ventilated patients. Chest. 1993;103:236-42.

26. Middleton RM III, Huff W, Brickey DA, Kirkpatrick MB. Comparison of quantitative cultures to semiquantitative loop cultures of bronchoscopic protected specimen brush samples. Chest. 1996; 109:1204-9.

27. Leal-Noval SR, Alfaro-Rodríguez E, Murillo-Cabeza F, Garnacho-Montero J, Rey-Pérez J, MuñozSánchez MA. Diagnostic value of the blind brush in mechanically ventilated patients with nosocomial pneumonia. Intensive Care Med. 1992;18:410-4.

28. Wood AY, Davit AJ II, Ciraulo DL, Arp NW, Richart CM, Maxwell RA, et al. A prospective assessment of diagnostic efficacy of blind protective bronchial brushings compared with bronchoscope-assisted lavage, bronchoscope-directed brushings, and blind endotracheal aspirates in ventilator-associated pneumonia. J Trauma. 2003; 55:825-34.

29. Torres A, Puig de la Bellacasa J, Rodríguez-Roisin R, Jiménez deAnta MT, Agusti-Vidal A. Diagnostic value of telescoping plugged catheters in mechanically ventilated patients with bacterial pneumonia using the Metras catheter. Am Rev Respir Dis.1988;138:117-20.

30. Kollef MH, Bock KR, Richards RD, Hearns ML. The safety and diagnostic accuracy of minibronchoalveolar lavage in patients suspected of ventilator-associated pneumonia. Ann Intern Med. 1995;122:743-8.

31. Levy H. Comparison of Ballard catheter bronchoalveolar lavage with bronchoscopic bronchoalveolar lavage. Chest. 1994;106:1753-8.

32. Timsit JF, Misset B, Renaud B, Goldstein FW, Carlet J. Effect of previous antimicrobial therapy on the accuracy of the main procedures used to diagnose nosocomial pneumonia in patients who are using ventilation. Chest. 1995;108:1036-40.

33. Solé Violán J, Rodríguez de Castro F, Caminero J, Bordes A, Manzano JL. Comparative efficacy of broncoalveolar lavage and telescoping plugged catheter in the diagnosis of pneumonia in mechanically ventilated patients. Chest. 1993;103:386-90.

34. Torres A, Martos A, Puig de la Bellacasa J, Ferrer M, el-Ebiary M, González J, et al. Specificity of endotracheal aspiration, protected specimen brush, and broncoalveolar lavage in mechanically ventilated patients. Am Rev Respir Dis. 1993;147:952-7.

35. Marik PE, Careau P. A comparison of minibronchoalveolar lavage and blind-protected specimen brush sampling in ventilated patients with suspected pneumonia. J Crit Care. 1998;13:67-72.

36. Sirvent JM, Vidaur L, González S, Castro P, de Batle $\mathrm{J}$, Castro A, et al. Microscopic examination of intracellular organisms in protected bronchoalveolar mini-lavage fluid for the diagnosis of ventilator-associated pneumonia. Chest. 2003;123:518-23.

37. De Jaeger A, Litalien C, Lacroix J, Guertin MC, Infante-Rivard C. Protected specimen brush or bronchoalveolar lavage to diagnose bacterial nosocomial pneumonia in ventilated adults: a meta-analysis. Crit Care Med. 1999;27:2548-60.

38. Dotson RG, Pingleton SK. The effect of antibiotic therapy on recovery of intracellular bacteria from bronchoalveolar lavage in suspected ventilator-associated nosocomial pneumonia. Chest. 1993;103:541-6.

39. Trouillet JL, Chastre J, Vuagnat A, Joly-Guillou ML, Combaux D, Dombret MC, et al. Ventilator-associated pneumonia caused by potentially drug-resistant bacteria. Am J Respir Crit Care Med. 1998; 157:531-9.

40. Fridkin SK, Lawton R, Edwards JR, Tenover FC, McGowan JE, Gaynes RP, et al. Monitoring antimicrobial use and resistance: comparison with a national benchmark on reducing vancomycin use and vancomycin-resistant enterococci. Emerg Infect Dis. 2002;8:702-7.

41. Chastre J. Evolving problems with resistant pathogens. Clin Microbiol Infect. 2008;14:3-14.

42. Rello J, Ausina V, Ricart M, Castella J, Prats G. Impact of previous antimicrobial therapy on the etiology and outcome of ventilator-associated pneumonia. Chest. 1993;104:1230-5.

43. Lynch JP. Hospital-acquired pneumonia. Risk factors, microbiology, and treatment. Chest. 2001;119:373S-84.

44. Ewig S, Bauer T, Torres A. The pulmonary physician in critical care. Nosocomial pneumonia. Thorax. 2002;57:366-71. 